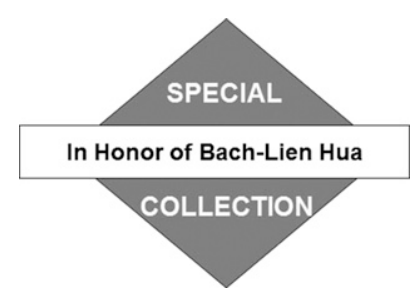

\title{
Tracer Stirring around a Meddy: The Formation of Layering
}

\author{
Thomas Meunier, Claire Ménesguen, Richard Schopp, and Sylvie Le GentiL \\ Laboratoire de Physique des Océans/Ifremer, Plouzané, Brittany, France
}

(Manuscript received 7 April 2014, in final form 30 October 2014)

\begin{abstract}
The dynamics of the formation of layering surrounding meddy-like vortex lenses is investigated using primitive equation (PE), quasigeostrophic (QG), and tracer advection models. Recent in situ data inside a meddy confirmed the formation of highly density-compensated layers in temperature and salinity at the periphery of the vortex core. Very high-resolution PE modeling of an idealized meddy showed the formation of realistic layers even in the absence of double-diffusive processes. The strong density compensation observed in the PE model, in good agreement with in situ data, suggests that stirring might be a leading process in the generation of layering. Passive tracer experiments confirmed that the vertical variance cascade in the periphery of the vortex core is triggered by the vertical shear of the azimuthal velocity, resulting in the generation of thin layers. The time evolution of this process down to scales of $O(10) \mathrm{m}$ is quantified, and a simple scaling is proposed and shown to describe precisely the thinning down of the layers as a function of the initial tracer column's horizontal width and the vertical shear of the azimuthal velocity. Nonlinear QG simulations were performed and analyzed for comparison with the work of Hua et al. A step-by-step interpretation of these results on the evolution of layering is proposed in the context of tracer stirring.
\end{abstract}

\section{Introduction}

Mediterranean water eddies (meddies) are coherent mesoscale features commonly observed in the North Atlantic. They are vortex lenses characterized by the contrast of hydrographic properties of the warm and salty Mediterranean water trapped within their core and the fresher and cooler surrounding water. This core, usually 650 to $750 \mathrm{~m}$ thick and centered near $1200 \mathrm{~m}$, has well-mixed water properties with typical temperature and salinity values ranging from respectively $11.5^{\circ}$ to $13.5^{\circ} \mathrm{C}$ and 36.2 to 36.8 psu (Armi and Zenk 1984; Schultz-Tokos and Rossby 1991; Pingree and Le Cann 1993; Prater and Sanford 1994; Paillet et al. 2002; Carton et al. 2010). The density anomaly associated with these temperature and salinity distributions consists of stretched isopycnals within the eddy core and squeezed isopycnals above and below. This results in a baroclinic tripole potential vorticity anomaly (PVA) structure, where a

Corresponding author address: Thomas Meunier, Laboratoire de Physique des Océans, Ifremer, ZI de la Pointe du Diable, Plouzané, 29280, France.

E-mail: thomas.meunier@ifremer.fr negative PVA core is surrounded by positive PVA lobes above and below (Carton et al. 2002; Paillet et al. 2002). This PVA distribution, largely dominated by vortex stretching (Morel and McWilliams 1997; Hua et al. 2013), induces an anticyclonic circulation, with a typical rotation period of 5 to 6 days (Armi et al. 1989; SchultzTokos and Rossby 1991). Even though they are strongly baroclinic structures, meddies were shown to have an appreciable barotropic signature (Jungclaus 1999). The typical azimuthal velocity found within eddy cores ranges from 15 to $50 \mathrm{~cm} \mathrm{~s}^{-1}$ (Armi and Zenk 1984; Pingree and Le Cann 1993; Schultz-Tokos et al. 1994; Carton et al. 2002) and is strongly vertically sheared (Schultz-Tokos and Rossby 1991). Within the radius of maximum velocity, they are close to solid body rotation (Schultz-Tokos and Rossby 1991). Typical meddies have a 15- to 35-km radius (Pingree and Le Cann 1993; Carton et al. 2002, 2010; Paillet et al. 2002), but a large range of sizes is reported in the literature with values ranging from 9 (Prater and Sanford 1994) to $50 \mathrm{~km}$ (Armi and Zenk 1984). The typical local Burger number in meddies is also quite variable, but is usually low with typical values of about 0.1 to 0.3 (Beckmann and Käse 1989; Pingree and Le Cann 1993; Morel and McWilliams 1997). 
Meddies detach from the Mediterranean undercurrent that flows along the Portuguese slope through baroclinic instability (Jungclaus 1999). Even though most attention has been paid so far to anticyclonic meddies, recent studies have shown that they were often linked to smaller cyclonic structures detaching simultaneously from the boundary current (Carton et al. 2002; Barbosa Aguiar et al. 2013). This detachment usually occurs in the vicinity of the major topographic features of the Iberian coast such as Cape São Vicente and the Estremadura promontory (Bower et al. 1997). They then drift southwestward and can travel more than $1000 \mathrm{~km}$ away from the eastern boundary and reach lifetimes of more than $2 \mathrm{yr}$ (Armi et al. 1989) if they do not crash against sea mounts and collapse (Schultz-Tokos et al. 1994; Richardson et al. 2000).

A substantial volume of Mediterranean water gets trapped in each meddy; a 50-km radius feature's transport is estimated to be equivalent to 10 days of Mediterranean outflow through the Gibraltar straight (Armi and Zenk 1984; Schultz-Tokos and Rossby 1991). As 15 to 20 meddies were observed to form in a year (Bower et al. 1997; Richardson et al. 2000), meddies carry most of the Mediterranean outflow and might therefore have an important climatic impact by having a crucial role in the redistribution of heat and salt into the North Atlantic. A net decrease of meddies' sizes and hydrographic anomalies with time (Hebert et al. 1990; Armi et al. 1989) along with the observation of "salty trails" (Song et al. 2011) suggest that meddies continuously shed Mediterranean water along their journey through the North Atlantic. Of particular interest is the understanding of how they release this thermodynamic content into the ocean.

Early in situ observations have revealed the presence of intrusions or interleavings of water masses in the periphery of meddies while their cores remained homogeneous. They are obvious in both temperature and salinity vertical profiles (Armi and Zenk 1984; Ruddick and Hebert 1988; Armi et al. 1989; Ruddick 1992; Pingree and Le Cann 1993; Ruddick and Walsh 1995). The typical vertical scale of these interleavings was shown to vary from 25 to $75 \mathrm{~m}$ (Armi and Zenk 1984; Ruddick 1992). Their essential role in the long-term decay of meddies was pointed out by Armi et al. (1989), who noted a net loss of heat and salt after a period of a few months, while the intrusion-affected zone had ended up reaching the meddy core. Ruddick and Hebert (1988) noticed a progress of the intrusions toward the eddy core of $30 \mathrm{~km}$ in $1 \mathrm{yr}$. The observation of an increased spindown over and below the meddy core by Schultz-Tokos and Rossby (1991) also suggests the dominant influence of these intrusions in the meddy decay.

Recent advances in water column seismic imagery allowed an unprecedented high-resolution mapping of

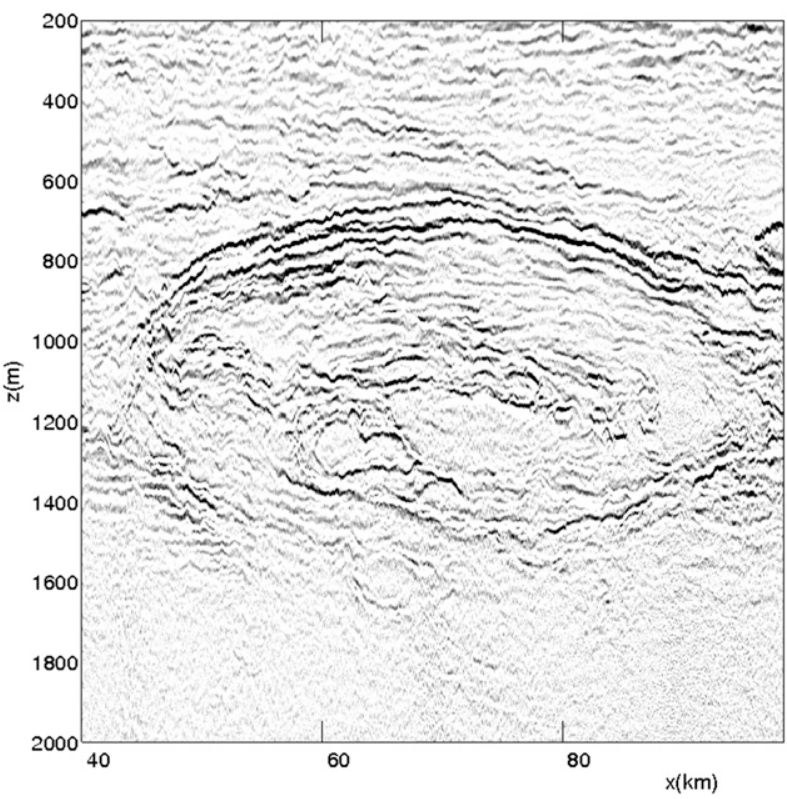

FIG. 1. Seismic reflectivity of the Meddy GO, adapted from Hua et al. (2013). The reflectors are doming all around the meddy, similar to Biescas et al. (2014).

these structures (10-15-m horizontal resolution) (Biescas et al. 2008; Ménesguen et al. 2009). The reflectivity of the seismic signal being directly linked to the vertical gradients of the temperature anomalies (Biescas et al. 2008; Ruddick et al. 2009), the vertical temperature anomalies associated with the interleavings appear clearly in the seismic imagery (see Fig. 1). These temperature anomalies materialize as a stacking of concentric layers exhibiting a striking lateral coherence.

This large lateral coherence has led recent authors to describe these elongated vertically stacked structures as layers or layering (see, e.g., Ménesguen et al. 2009). From now on, the latter terminology will be used in this paper.

The typical vertical scale of layering was observed to range from 20 to $75 \mathrm{~m}$, consistent with CTD observations (Quentel et al. 2010), while their horizontal scale can reach up to the vortex radius (Biescas et al. 2008; Ménesguen et al. 2009). Hua et al. (2013) investigated the production of layering using a high-resolution quasigeostrophic (QG) model and revealed for the first time its three-dimensional structure. They highlighted the threedimensional helical patterns forming above and below the meddy core, introducing the idea that layering would not consist of a simple stacking of pancake-like structures. They also noted that layering formation was associated with a direct cascade of energy toward dissipative scales. The increased variance of tracer and momentum leading to an increase of diffusion and dissipation could 
explain the correlation between layering and meddy decay observed by Schultz-Tokos and Rossby (1991).

The dynamics underlying the formation of layering is still an open question. A review of some of the possible processes can be found in Song et al. (2011). While most of the early studies attributed this phenomenon to doublediffusive processes (Ruddick and Hebert 1988; Armi et al. 1989; Hebert et al. 1990; Pingree and Le Cann 1993; Ruddick and Walsh 1995), Ruddick (1992) intuited that a larger-scale vertically sheared flow could bring the temperature and salinity anomalies into the scale where diffusion becomes important. More recent works, made possible by the improved spatial resolution of these layered structures in seismic imagery, have emphasized this possible contribution of stirring (Ruddick et al. 2009; Pinheiro et al. 2010; Song et al. 2011) in the vertical cascade of the thermohaline gradients, though they argued that double-diffusive processes were still likely to play an important role at the smallest vertical scales. However, Hua et al.'s (2013) recent QG numerical simulations, where the salinity variable is not taken into account, suggested a new process for the generation of fully developed layering and the cascade of vertical scales down to a few meters. Previous linear results from Nguyen et al. (2012), showing the existence of critical layers (the locus where the azimuthal velocity equals the phase speed of the growing perturbation) in the periphery of vortex cores associated with low azimuthal modes' baroclinic instability, led Hua et al. (2013) to discuss the link between layering and critical layers. They found that layering was principally forming in the vicinity of these critical layers and argued that layering was thus consistent with a windup of potential vorticity around the latter in a threedimensional analog of Balmforth et al.'s (1998) process.

Despite new insights brought by the recent works of Song et al. (2011) and Hua et al. (2013) in both the description and understanding of the layering phenomenon, some particular points remain unclear or need further investigation. In particular, the hypothesis of layering being entirely driven by critical layer instability becomes ambiguous once the instability has grown enough to leave the linear regime; critical layers can only be analyzed when perturbations are small enough so that the mean flow can be considered as nearly steady. Unlike Balmforth et al. (1998), who used neutral modes to study the rolling of PV around critical layers, in the case of an unstable meddy such as Hua et al.'s (2013), the perturbations grow so that once the mode-2 instability breaks into spiral arms critical layers would vanish. One would then expect the layers to mix and decay right after the linear stage of the perturbation growth, while they keep on intensifying and sharpening.

In the present paper, we wish to further investigate one of the hypotheses of Song et al. (2011), who intuited that tracer stirring by the mean flow could be an effective process to sharpen the gradients at the periphery of meddies, similar to front sharpening and downward cascade of variance near frontal structures described by several authors (MacVean and Woods 1980; Woods et al. 1986; Haynes and Anglade 1997; Klein et al. 1998). We also wish to discuss the results from Hua et al. (2013) and try to give more details on the respective role of baroclinic instability and stirring in their nonlinear QG model. This study is based on various frameworks, including passive tracer advection, primitive equation (PE), and QG modeling as well as in situ data.

The paper is organized as follows. In section 2, the physical and numerical frameworks are described: the parameterization of the vortex lens is explained (section 2a) and the details of the PE, QG, and tracer models used are presented, respectively, in sections $2 \mathrm{~b}, 2 \mathrm{c}$, and $2 \mathrm{~d}$. Section 3 focuses on the density compensation of the thermohaline anomalies observed in situ in a meddy (section 3a) and simulated in the PE model (section 3b), which suggests a possibly predominant role of tracer stirring in the generation of layering. The latter hypothesis is studied in detail in section 4 , where tracer experiments using a steady mean flow are performed and analyzed, showing a great similitude with the observed and modeled layers. These results are used to discuss in detail the generation of layering as observed in a nonlinear QG model by Hua et al. (2013) (section 5). Last, a summary of the main conclusions of this study can be found in section 6 .

\section{Physical and numerical setup}

\section{a. Parameterization of the vortex lens}

Meddies are characterized by a negative PVA core associated with stretched isopycnals, surrounded above and below by two positive PVA lobes associated with squeezed isopycnals. PVA distribution as well as the aspect ratio are key parameters for vortex stability properties including unstable mode selection and growth rates. A Lorentzian streamfunction as proposed by Hua et al. (2013) to fit the PVA distribution of typical meddies is used all along in the present work:

$$
\begin{aligned}
\psi & =\tilde{\psi}_{0}\left(1+d^{2}+d^{4}\right)^{-1}, \quad \text { and } \\
d & =\sqrt{\left(\frac{r}{L}\right)^{2}+\left(\frac{z}{H}\right)^{2}}
\end{aligned}
$$

where $r$ is the horizontal distance from the rotation axis, $L$ is the vortex radius, $z$ is the vertical distance from the vortex center, and $H$ is the vortex half thickness. The velocity field is strictly azimuthal. It is sheared both in 

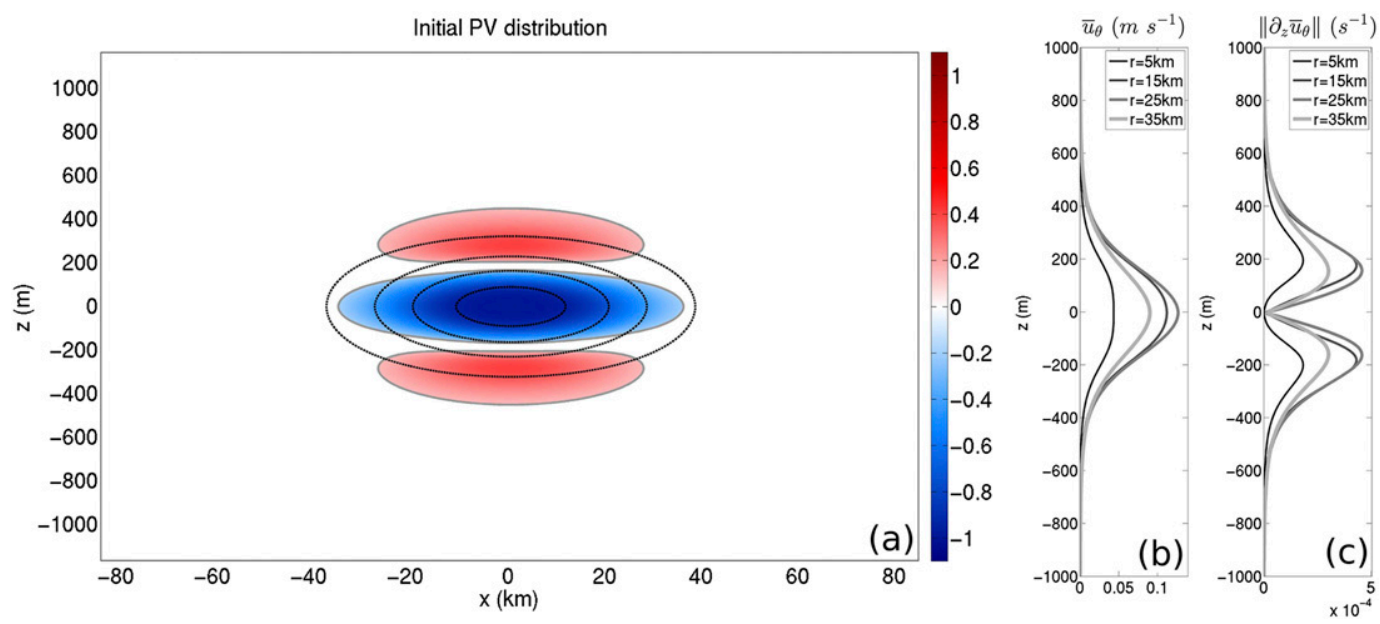

FIG. 2. (a) PVA distribution of the meddy-like vortex lens (nondimensional). The thick gray lines represent the -0.2 and 0.1 PVA contours, while the black dotted lines represent the associated streamfunction contours. (b) Vertical profiles of the azimuthal velocity at 5, 15, 25, and $35 \mathrm{~km}$ from the vortex rotation axis. (c) As in (b), but for the absolute value of the vertical shear of the azimuthal velocity.

the radial and vertical directions. The shape of the vortex is entirely determined by its aspect ratio $\delta=H / L$ and its intensity by the factor $\tilde{\psi}_{0}$. All simulations are performed using constant values of the Brunt-Väisälä frequency $\left\{N_{0}^{2}=-\left(g / \rho_{0}\right)[d \bar{\rho}(z) / d z]\right.$, where $\bar{\rho}(z)$ is the mean density, and $g$ is the gravity acceleration $\}$ and Coriolis parameter $\left(f_{0}=2 \Omega \sin \theta\right.$, where $\Omega$ is the rotation rate of Earth and $\theta$ is the latitude). The aspect ratio is thus linearly linked to a local Burger number $\mathrm{Bu}=\left(N_{0}^{2} / f_{0}^{2}\right) \delta^{2}$. The following parameter values are chosen to fit typical values of radius, thickness, stratification, and PVA distribution: $N_{0}=3.2 \times 10^{-3} \mathrm{~s}^{-1}, f_{0}=8 \times 10^{-5} \mathrm{~s}^{-1}, \mathrm{Bu}=$ 0.1 , and $L=35 \mathrm{~km}$. The small value of the chosen local Burger number is consistent with a typical meddy aspect ratio with PVA largely dominated by vortex stretching. A PVA cross section of the vortex used in the simulations is shown in Fig. 2a as well as vertical profiles of the azimuthal velocity (Fig. 2b) and the absolute value of the vertical shear of the azimuthal velocity (Fig. 2c) at 5, 15,25 , and $35 \mathrm{~km}$ from the vortex rotation axis.

\section{b. The PE numerical model}

In section 3b, we use the numerical model of Aiki and Yamagata (2006) (see also Aiki et al. 2011) in which the hydrostatic and incompressible Boussinesq equations are implemented on the $f$ plane. In the present work, we use a flat bottom configuration with slippery conditions at the bottom, a free surface with implicit scheme (Dukowicz and Smith 1994), and open radiative lateral boundary conditions. The advection scheme is a thirdorder upwind scheme for momentum and tracers that prevents spurious oscillations. Vertical momentum dissipation and tracer diffusivity are both modeled by a biharmonic operator with a coefficient of $1.25 \times$ $10^{-5} \mathrm{~m}^{4} \mathrm{~s}^{-1}$ for a vertical grid step of $5 \mathrm{~m}$. The use of the same diffusion coefficient for temperature and salinity inhibits the occurrence of any double-diffusive process. The time integration is done using a leapfrog scheme with the Matsuno scheme blended every 20 time steps. No explicit forcing is applied.

\section{c. The quasigeostrophic numerical model}

The model used is Hua and Haidvogel's (1986) fully spectral QG model. It is run with horizontal and vertical grid steps of $d x=500 \mathrm{~m}$ and $d z=6.25 \mathrm{~m}$ in a doubly periodic $250-\mathrm{km}$-wide and 2400-m-deep parallelepiped domain. The time step is $d t=100 \mathrm{~s}$. Even though this resolution is slightly coarser than Hua et al.'s (2013), sensitivity tests showed that it is high enough for the occurrence of well-developed layering around the vortex. The model consists of a simple integration of the quasigeostrophic potential vorticity (QGPV) conservation equation:

$$
\partial_{t} q+J(\psi, q)=\nabla^{6} \psi
$$

where $\psi$ is the streamfunction, $J$ is the Jacobian operator, and $q$ is the QGPV defined as

$$
q=\nabla^{2} \psi+\partial_{z}\left(\frac{f_{0}^{2}}{N_{0}^{2}} \partial_{z} \psi\right),
$$

where $\nabla^{2}$ is the $2 \mathrm{D}$ horizontal Laplacian operator. The biharmonic operator $\nabla^{6} \psi$ is included to avoid numerical stability issues and is chosen to be as small as possible. Time integration of Eq. (3) requires the inversion of 
QGPV to compute the advection terms (i.e., the Jacobian operator). This operation is performed in the Fourier space where integration of the Laplacian comes to a simple multiplication by the squared wavenumber. A decomposition into vertical modes is also performed in the model.

\section{d. The tracer advection model}

To study the advective properties of the vortex lens, tracer experiments were performed using the stationary mean flow field described by Eq. (1). Advecting the tracer in a stationary velocity field forbids any dynamical process from taking place. It allows us to study the effect of the mean flow on the tracer from a kinematic point of view.

The tracer model used is based on the tangent linear version of the QG model described in the last section. It solves the equation

$$
\partial_{t} q^{\prime}+J\left(\bar{\psi}, q^{\prime}\right)+J\left(\psi^{\prime}, \bar{q}\right)=0
$$

where the overbar denotes the steady mean flow and the primes denote the perturbations. Dropping the term $J\left(\psi^{\prime}, \bar{q}\right)$ from Eq. (5), the QG tangent linear model becomes a tracer conservation model in a mean flow. In that case, the velocity field $(\bar{u}, \bar{v})$ of the base state remains constant in time. As vertical velocities inside a meddy are weak and layering was shown to appear in QG models with no vertical advection (Hua et al. 2013), the advective field chosen here is purely horizontal. The conservation equation of a passive tracer with concentration $\chi$ reads

$$
\partial_{t} \chi+J(\bar{\psi}, \chi)=0
$$

where $\bar{\psi}$ is the base state streamfunction.

Various initial tracer distributions were used in this study. The reference experiment was run with a horizontally random barotropic tracer distribution, while sensitivity experiments were carried out using regularly spaced cylindrical tracer columns. The sensitivity tests included variation of the columns' radii as well as their distance from the vortex rotation axis.

\section{Temperature and salinity anomalies compensated in density: An indication of tracer stirring}

\section{a. Analysis of CTD profiles inside a typical meddy}

CTD profiles from the Geophysical Oceanography (GO) field survey were analyzed to characterize the vertical structure of temperature and salinity anomalies in a meddy. The GO experiment, carried out in 2007 in the Gulf of Cádiz, is a joint seismic and oceanographic experiment originally designed to study meddies' fine structure (Hobbs 2007).

Figure 3a shows a profile of density anomaly $\left(\rho^{\prime} / \rho_{0}\right)$ and the associated contributions of temperature $\left(\beta S^{\prime}\right)$ and salinity $\left(\alpha T^{\prime}\right)$ in the vicinity of the rotation axis. The parameter $\alpha$ is the thermal expansion coefficient, $\beta$ is the saline contraction coefficient, $T^{\prime}$ is the temperature anomaly, and $S^{\prime}$ is the salinity anomaly. These profiles have been high-pass filtered in order to exhibit vertical scales smaller than $200 \mathrm{~m}$.

Even though some small-scale layering is noticeable in the density anomaly signal, much larger amplitudes are obvious in the salinity and temperature contributions. The compensation of the latter two in the density anomaly field is considerable (up to $90 \%$ ).

The spice variable is a useful quantity in the study of density-compensating water masses. It is defined as $\tau=\alpha T+\beta S$ (Veronis 1972; Munk 1981). It is a Lagrangian tracer. Significant variability in spice associated with weak variability in density is an indicator of strongly "density-compensating" effects of temperature and salinity, and spice then behaves as a passive tracer.

The striking difference between the anomalies of spice and density can be inferred from Fig. 3b. While an important variability is obvious in the spice variable above and below the meddy core, the density shows little vertical variations, suggesting that the main physics working behind this layering phenomenon is consistent with tracer stirring.

\section{b. Evidences of compensated thermohaline layering in a PE model}

The PE model was integrated over 300 days. The time evolution of the vortex in terms of Ertel's PV is similar to the evolution of QGPV in Hua et al.'s (2013) QG simulations and will thus only be briefly recalled in this paragraph. The reader can refer to section 5 of this paper or to Hua et al. (2013) for an extended description. After about 30 days of integration, an azimuthal mode- 2 perturbation starts to grow until it eventually breaks after day 160 into two smaller satellite vortices. The vortex core then evolves progressively into an axisymmetric state, while the lobes remain in a nearly turbulent state, undergoing strong filamentation until the end of the simulation. To make easier the comparison with seismic imagery, simulated acoustic reflectivity images were built from the model outputs. As pointed out by Nandi et al. (2004) and Ruddick et al. (2009), reflectivity is mostly governed by the temperature vertical gradients. The simulated acoustic reflectivity inferred from $T$ and $S$ outputs reveal the formation of well-defined, vertically stacked layers, as shown in the vertical section in Fig. 4a. 

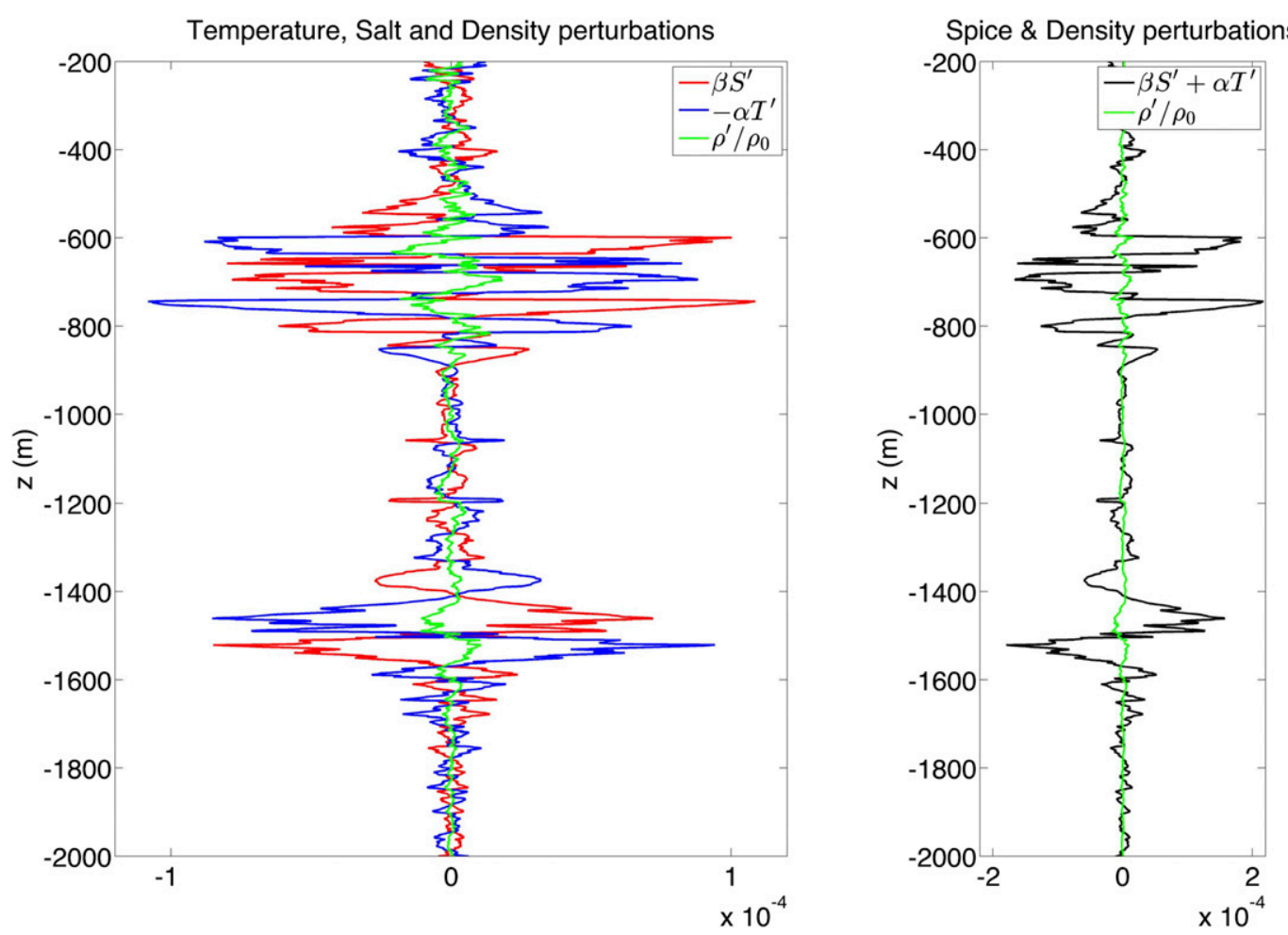

FIG. 3. (a) Contribution of temperature (blue) and salinity (red) in the density perturbation (green) near a meddy rotation axis. (b) Associated spice (black) and density (green) perturbation. Layering in tracer fields is observed above and below the meddy. Salinity effects on density compensate temperatures at $90 \%$ in the filtered density field. $T, S$, and the density have been filtered with a high-pass filter $(<200 \mathrm{~m})$.

Layers are also obvious in the spice section (Fig. 4b), while they are hardly discernible in the density field (not shown).

Figure 5a shows vertical profiles of density anomaly (green line) along with the contribution of temperature (blue curve) and salinity (red curve). These profiles are strikingly similar to the observed profiles within the meddy GO discussed in the last section. Layering is obvious above and below the vortex core as strong fluctuations in the temperature and salinity profiles. Density-compensating effects of the temperature and salinity anomalies can be inferred from the antisymmetry between the blue and red curves. As a result, while the density shows almost no anomaly, the spice profile is strongly fluctuating (Fig. 5b). Note that in the model, salinity and temperature have the same diffusion coefficient, thus inhibiting double-diffusive processes. This simplifying hypothesis used in the model could be the reason for the enhancement of density compensation (about 99\%) in the model over the data (90\%). However, even though double-diffusive processes are not explicitly studied here, the ability of a model that does not allow double diffusion to reproduce the main patterns of the observations suggests that stirring might be the leading process in the layering formation. Doublediffusive processes could, however, account for the observed difference.

\section{Tracer stirring in the steady vortex velocity field}

The results of the PE model shown in the last section exhibit the important density compensation of temperature and salinity in those layers, suggesting that temperature nearly behaves like a passive tracer. A simple and rigorous way of studying the stirring of such a tracer consists in modeling its advection in a stationary velocity field.

The tracer advection model is first initialized using a horizontally random, barotropic tracer distribution.

Figures 6 and 7 describe the evolution of the tracer properties after, respectively, 5 and 22 days of model integration. Figures $6 \mathrm{a}$ and 7 a show vertical sections of the tracer concentration; Figs. $6 \mathrm{~b}$ and $7 \mathrm{~b}$ show vertical sections of the tracer vertical gradient on which vertical sections of the horizontal streamlines were superimposed as red contours; Figs. $6 \mathrm{c}$ and $7 \mathrm{c}$ show vertical 

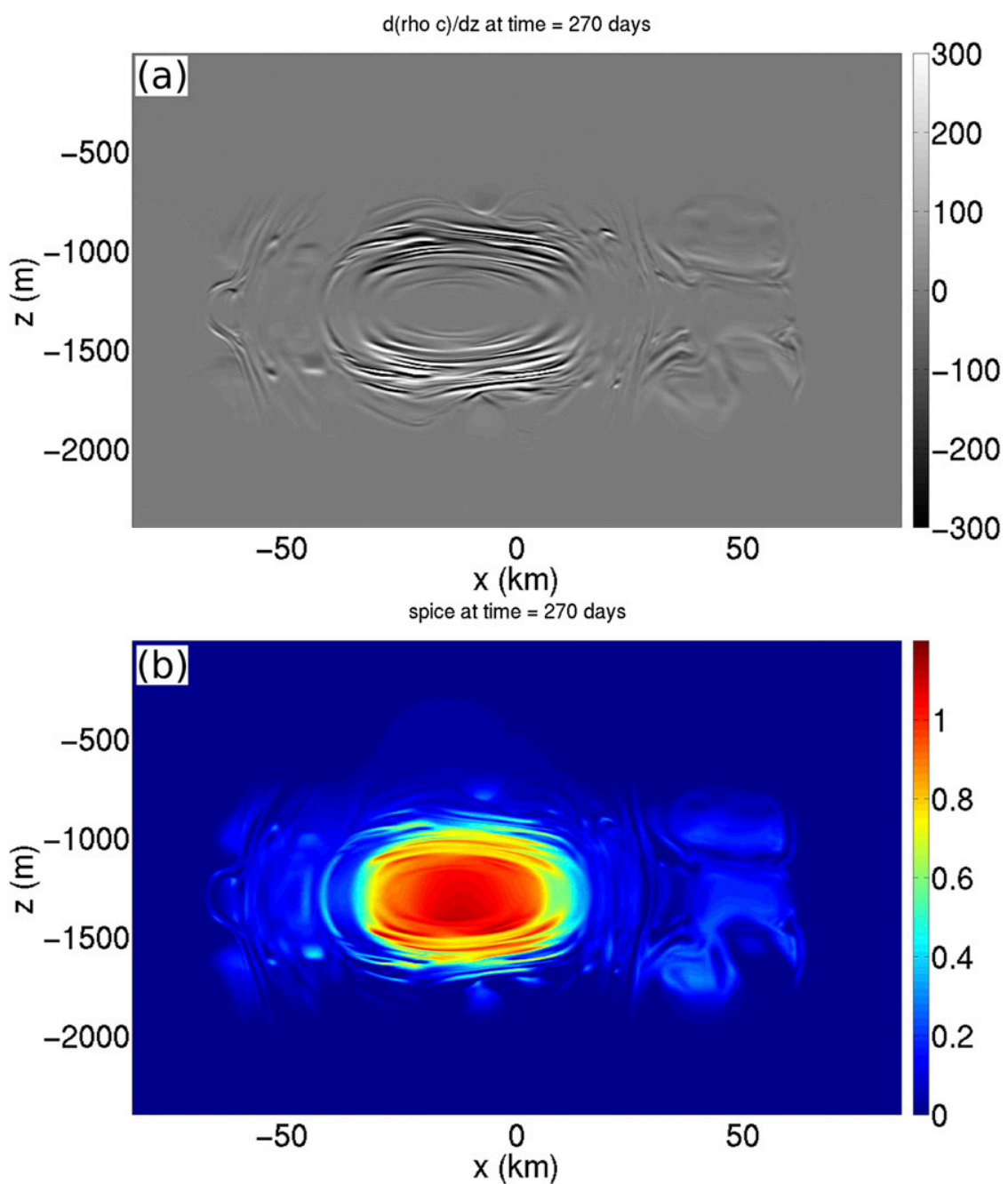

FIG. 4. (a) Vertical section of acoustic reflectivity in the central part of the vortex after 270 days of PE model integration. (b) As in (a), but for the spice variable. Layering is obvious both in acoustic reflectivity and spice.

tracer profiles at $0,12.5$, and $25 \mathrm{~km}$ from the eddy rotation axis; and Figs. $6 \mathrm{~d}$ and $7 \mathrm{~d}$ show the vertical tracer anomaly spectra $25 \mathrm{~km}$ away from the eddy rotation axis. The baroclinization of the tracer field is obvious from the first days of model integration as the tracer gradient quickly aligns with the streamfunction. The vertical variability is concentrated in the periphery of the vortex, where the vertical shear of the azimuthal velocity is at maximum (Fig. 2c), while the core, where there is no shear, remains homogeneous all along the experiment (Figs. 6a, 7a). The tracer and tracer vertical gradient organize as well-defined concentric layers spawning from the direct periphery of the eddy core toward the outside. Simultaneously, the length scales of those layers keep on thinning down with a spectral peak getting tighter (Figs. 6d, 7d). After 5 days of model run, the most energetic length scale is of about $35 \mathrm{~m}$, whereas after 22 days it has lowered down to nearly $15 \mathrm{~m}$. The amplitude of the tracer anomaly simultaneously increases (Fig. 7c).

A second experiment was designed to allow a simple interpretation and quantification of this process. The tracer distribution now consists of four barotropic columns equally spaced at $20 \mathrm{~km}$ from the eddy rotation axis. Figure 8 shows a 3D view of the tracer columns' deformation. The velocity field is plotted as blue arrows. It is strictly azimuthal. It is sheared both in the radial and vertical direction. At a given radius, the velocity increases vertically from outside toward the eddy core, the maximum shear being localized at about $200 \mathrm{~m}$ from the eddy center between the core and the lobes where PVA changes sign (Figs. 2b,c). This vertical shear induces 

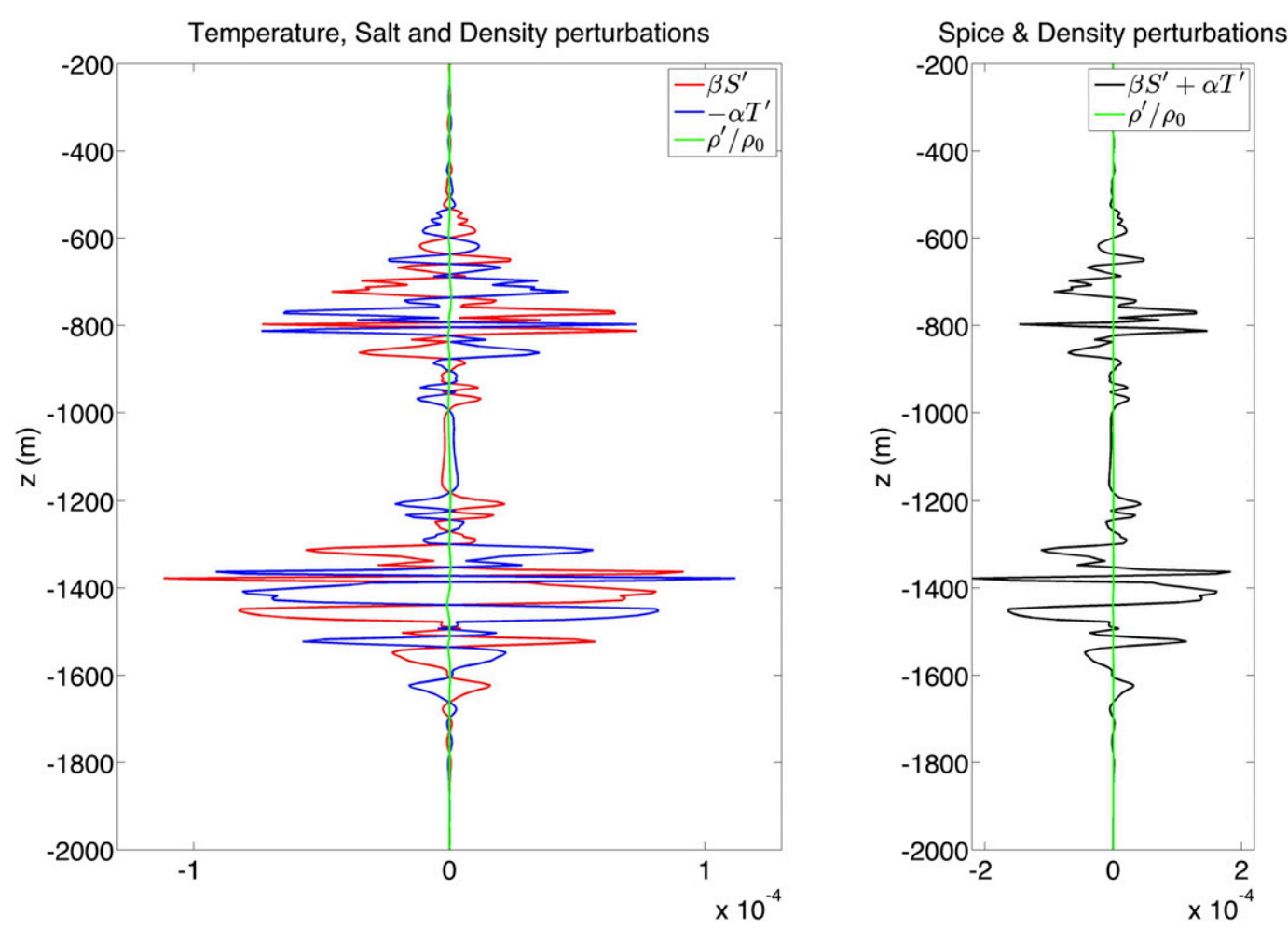

FIG. 5. As in Fig. 3, but for the modeled meddy. Strong anomalies in temperature and salinity result in strong spice anomalies while the density is almost unaffected.

a differential advection of the tracer tube, yielding an intense deformation of the tubes where velocity is high and a weaker deformation where the velocity is low. It results in a vertical tilting of the tracer columns whose signature is the layerlike vertical distribution as observed in Figs. 4, 6, and 7.

These two experiments show that the meddy's vertically sheared velocity field intrinsically transforms horizontal variability in the tracer field into vertical variability.

This transformation of horizontal into vertical gradients can be discussed following Haynes and Anglade's (1997) and Klein et al.'s (1998) approach, defining the local wave vector of the tracer distribution: $\mathbf{k}=\chi^{-1} \nabla \chi$, where $\chi$ is the tracer concentration. In cylindrical coordinates using the orthogonal base, where $\left(\mathbf{e}_{r}, \mathbf{e}_{\phi}, \mathbf{e}_{z}\right)$ are the unit vectors, the tracer conservation equation [Eq. (6)] reads

$$
\partial_{t} \chi+\frac{\bar{u}_{\phi}}{r} \partial_{\phi} \chi=0
$$

where $\bar{u}_{\phi}$ is the azimuthal velocity, and $r$ is the radial distance from the vortex rotation axis.

From Eq. (7) and its gradient, the evolution of the wave vector can be derived and depends entirely on the velocity gradient tensor A (Weiss 1991):

$$
\frac{d \mathbf{k}}{d t}=\mathbf{A} \cdot \mathbf{k}
$$

One can project Eq. (8) on the cylindrical base:

$$
\begin{aligned}
\partial_{t} k_{\phi}+\frac{\bar{u}_{\phi}}{r} \partial_{\phi} k_{\phi} & =0 \\
\partial_{t} k_{r}+\frac{\bar{u}_{\phi}}{r} \partial_{\phi} k_{r} & =\left(\frac{\bar{u}_{\phi}}{r}-\partial_{r} \bar{u}_{\phi}\right) k_{\phi}, \quad \text { and } \\
\partial_{t} k_{z}+\frac{\bar{u}_{\phi}}{r} \partial_{\phi} k_{z} & =-\partial_{z} \bar{u}_{\phi} k_{\phi},
\end{aligned}
$$

where $k_{\phi}=\mathbf{k} \cdot \mathbf{e}_{\phi}, k_{r}=\mathbf{k} \cdot \mathbf{e}_{r}$, and $k_{z}=\mathbf{k} \cdot \mathbf{e}_{z}$ are the wavenumbers in cylindrical coordinates.

Equation (9) shows that $k_{\phi}$ is simply advected along a closed streamline, as in the Rhines and Young's (1983) example of tracer stirring around a barotropic vortex. If $k_{\phi}^{0}(r, \phi)$ is the original wavenumber of the tracer distribution at $t=0$, the solution of this one-dimensional advection equation is thus $k_{\phi}(r, \phi, z, t)=k_{\phi}^{0}\left[r, \phi-\left(\bar{u}_{\phi} / r\right) t\right]$. It follows from Eqs. (10) and (11) that the evolution of the radial and vertical wavenumbers are independent from each other and only depend on the azimuthal wavenumber and velocity shear in their respective directions. 

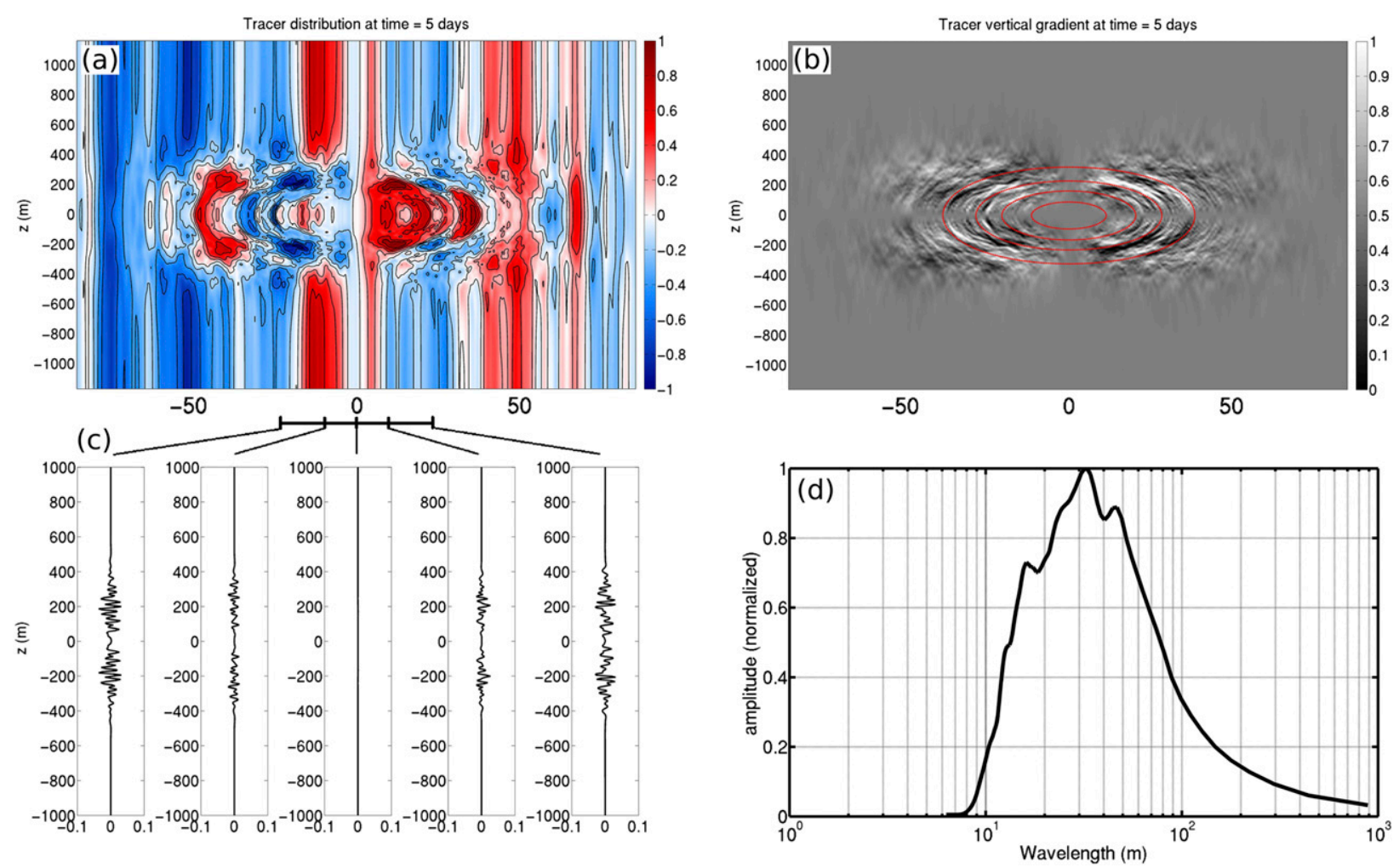

FIG. 6. Tracer properties after 5 days of model integration: (a) vertical section of the tracer concentration (normalized); (b) vertical section of the tracer's vertical gradient (normalized); (c) vertical profiles of tracer anomaly; and (d) spectral distribution of the tracer vertical anomaly. The baroclinization of the tracer field is evident from the first days of model integration.

The layering of the tracer in the vertical direction is thus entirely determined by the initial azimuthal distribution of the tracer and the vertical shear of the azimuthal velocity. Still following Klein et al. (1998), one can define the aspect ratio of the tracer gradient $\gamma=k_{z} / k_{\phi}$. Using Eqs. (9) and (11), its time evolution reads

$$
\frac{d \gamma}{d t}=-\partial_{z} \bar{u}_{\phi}
$$

The evolution of the aspect ratio of vertical to azimuthal variability is thus entirely determined by the vertical velocity shear.

While in Haynes and Anglade's (1997) and Klein et al.'s (1998) studies the horizontal tracer gradient is increasing at an exponential rate, preconditioning the vertical cascade, here the vertical gradient enhancement is only linked to the initial azimuthal gradient through the vertical shear of the azimuthal velocity. This difference arises from the absence of normal strain in the case of an axisymmetric vortex with zero radial velocity. A direct comparison with the latter author's work can be made considering a parallel shear flow in Euclidean coordinates: $[u=f(y, z), v=0]$. The velocity gradient matrix is degenerated because of the absence of normal strain:

$$
\mathbf{A}=\left(\begin{array}{lll}
\partial_{x} u & \partial_{x} v & 0 \\
\partial_{y} u & \partial_{y} v & 0 \\
\partial_{z} u & \partial_{z} v & 0
\end{array}\right)=\left(\begin{array}{ccc}
0 & 0 & 0 \\
\partial_{y} u & 0 & 0 \\
\partial_{z} u & 0 & 0
\end{array}\right)
$$

In that particular case, the wavenumber $k_{x}$ is simply advected as in Eq. (9), while the time evolutions of $k_{y}$ and $k_{z}$ are directly linked to $k_{x}$ through the velocity shear in their respective direction.

Following this simple example, it is possible to build a linear scaling to infer an approximate rate of transfer of horizontal to vertical variability at a given position inside the eddy. Considering a tracer tube of radius $L_{\phi}$ in a linearly sheared, two-dimensional velocity field $\bar{u}(z)=\sigma\left(z-z_{0}\right)$, at a given time $t$ and $z$ level, the tracer tube has undergone an inclination of $\beta=\arctan (\sigma t)$ (Fig. 9). Defining the layer thickness $\epsilon$ as the vertical distance between the two opposite edges of the tracer column, it thus evolves as

$$
\epsilon=\frac{2 L_{\phi}}{\sigma t} .
$$



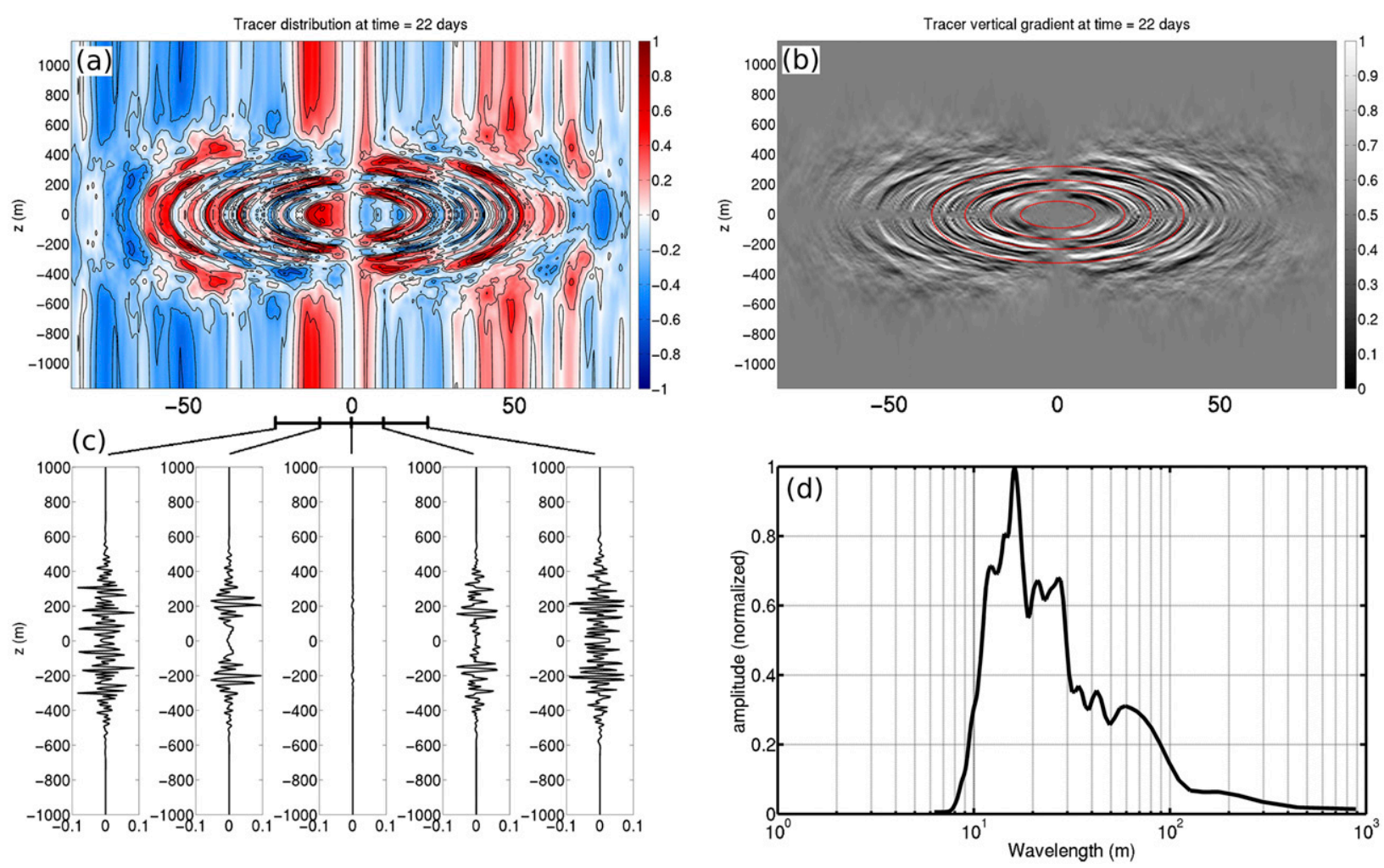

FIG. 7. As in Fig. 6, but after 22 days of model integration. Layering has increased with time, while the vertical length scale has decreased.

To quantify this process and compare the modeled rate of transfer from horizontal into vertical variability with the scaling, sensitivity experiments were carried out by varying the distance of the tracer tubes from the eddy center (and thus the vertical shear) in the initial velocity field.

Figure 10 shows the time evolution of the layer thickness as inferred from the above-proposed scaling for various values of $\sigma$ corresponding to the maximum vertical shear at a radial distance from the vortex center of $5,25,35$, and $50 \mathrm{~km}$ and a tracer tube width of $10 \mathrm{~km}$. The circled stars are the thicknesses from the corresponding model runs. The striking agreement between the modeled and scaled values of $\epsilon$ shows that the formation of layering in a tracer field can be directly related to the vertical shear of the horizontal velocity field of the meddy. This good agreement between model outputs and theory in the case of the tracer model suggests that the same process could as well be important in models with more complex dynamics.

The above discussion focused on the evolution of the vertical tracer gradients. This restricted point of view is sufficient for the interpretation of seismic images that show vertical temperature gradients. It is also appropriate for a further discussion on the study of Hua et al.
(2013) that is largely based on vortex stretching analysis (also related to a vertical derivative).

However, as shown in Eq. (10), stirring in the radial direction (in the sense of cylindrical coordinates) is also at work and will act to enhance the radial tracer gradient $\partial_{r} \chi$. It is possible to build a more general tracer gradient combining both the effects of vertical and radial shears. Normalizing the vertical coordinates by the vortex aspect ratio $\tilde{z}=z / \delta$, the vortex lens becomes a sphere. One can then switch from this stretched cylindrical coordinate system $r, \phi, z$ to a spherical coordinate system $R, \phi, \theta$, where $R=\sqrt{r^{2}+\tilde{z}^{2}}$ and introduce the generalized radial gradient:

$$
\nabla \chi \cdot \mathbf{e}_{R}=\sqrt{\left(\nabla \chi \cdot \mathbf{e}_{r}\right)^{2}+\left(\nabla \chi \cdot \mathbf{e}_{\tilde{z}}\right)^{2}} .
$$

Figure 11 shows the distribution of the tracergeneralized radial gradient after 22 days of integration. The layers appear not to be vertically stacked as was the vertical gradient in Fig. $7 \mathrm{~b}$, but to be concentric and located all around the vortex periphery. A striking feature is the near isotropy of those layers in stretched coordinates. This shows that horizontally stacked layers might exist in the lateral periphery of meddies in the real ocean and that they should have an approximate ratio of 

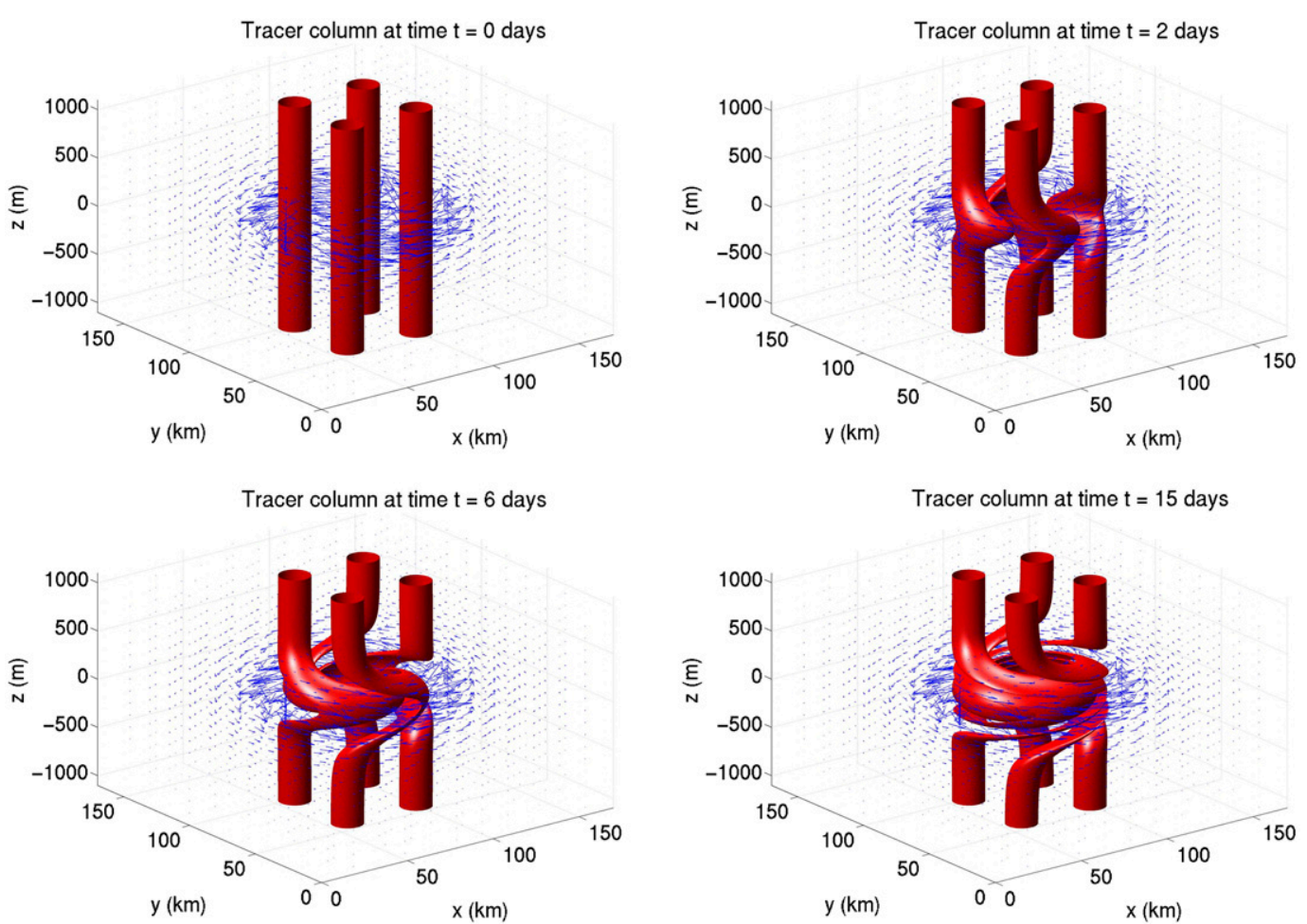

FIG. 8. 3D view of the tracer columns deformation at time $=0,2,6$, and 15 days in the tangent linear model. The blue arrows are the velocity vectors. An intense differential winding of the tracer tubes by the vortex velocity field yields to the formation of vertical variability.

$\delta$ with the thickness of the observed vertically stacked layers. However, note that meddies are almost flat features, with $\delta=O\left(10^{-2}\right)$, so the vertically stacked layers remain the most obvious structures associated with the layering process.

\section{Discussion on the nonlinear QG simulations}

The stirring properties of a modeled meddy described in the last section suggest a further interpretation of Hua et al.'s (2013) results. The nonlinear QG model described in section 2c was integrated for over 300 days. The initial velocity field is the same as for the PE and tracer experiments. Despite the slightly coarser resolution of our configuration compared to Hua et al.'s (2013), the time evolution of the vortex is similar.

Figure 12 shows horizontal sections of QGPV in the lobes (Figs. 12a,e) and in the core (Figs. 12b,f), vertical sections of QGPV in the center of the vortex (Figs. 12c,g) and vertical profiles of high-pass filtered QGPV at 0 , 12.5, and $25 \mathrm{~km}$ from the initial vortex center (Figs. 12d,h) after 57 and 240 days of model integration. A modal analysis was also performed to help discussion and is shown in Fig. 13. The QGPV and streamfunction are projected into a base of azimuthal modes $m:[\psi, q](r, \phi, z, t)=$ $\sum\left[\psi_{m}, q_{m}\right](r, z, t) e^{i m \phi}$.

The vortex evolution can be decomposed into three main stages: The linear stage (A), the splitting stage (B), and the restabilized stage (C). During the first 100 days of integration (stage A), the vortex undergoes baroclinic instability as the symmetric $(m=2)$ and antisymmetric

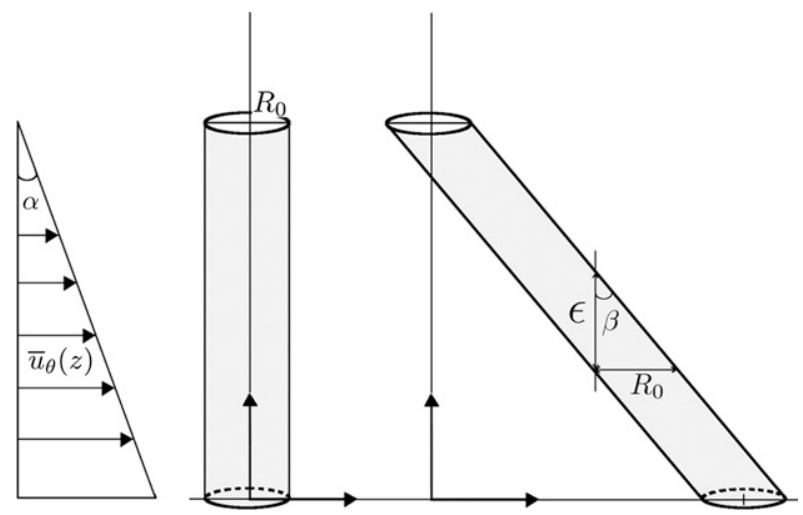

FIG. 9. Schematic view of a tracer tube deformation in a linearly sheared flow. The $\epsilon$ parameter is the vertical distance between two isolines of tracer, $R_{0}$ is the diameter of the tracer column, and the velocity shear can be defined by the angle $\alpha=\arctan (\sigma)$. The inclination of the tracer tube is defined by the angle $\beta=\arctan (\sigma t)$. 


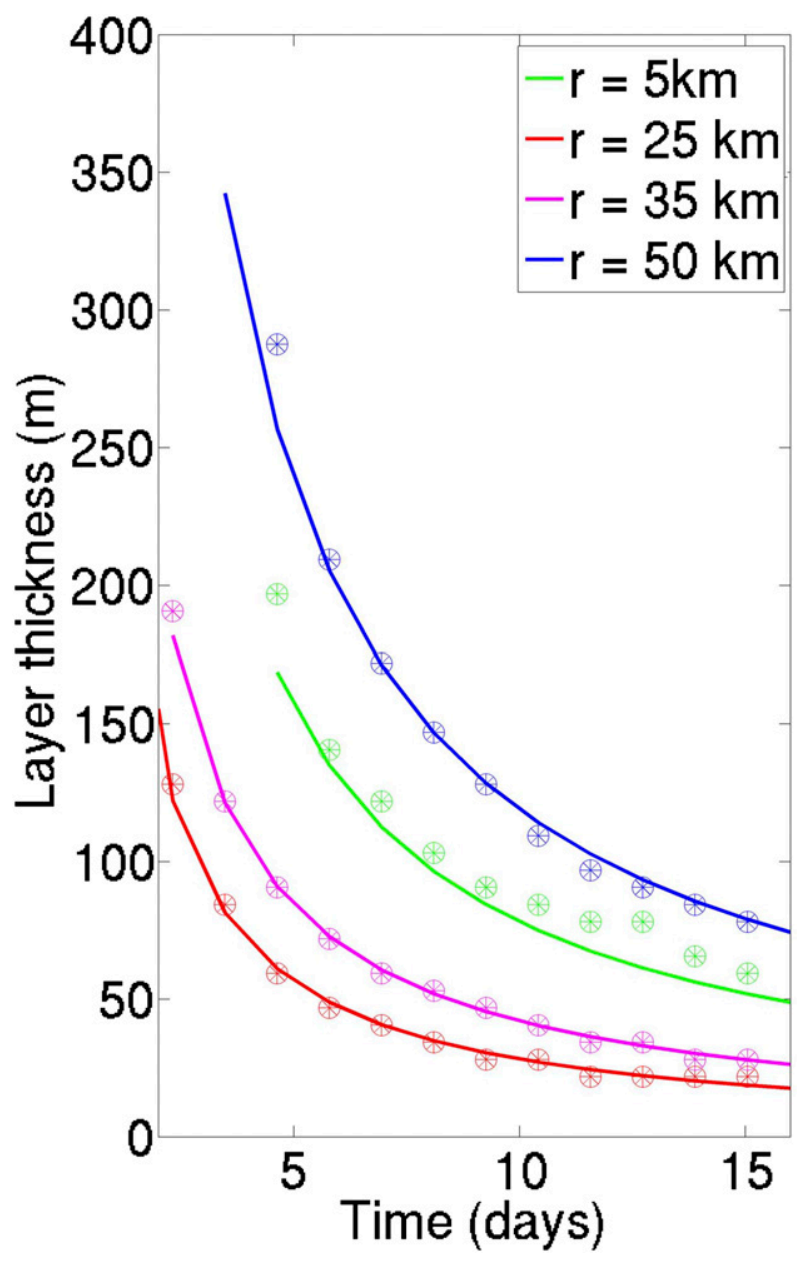

FIG. 10. Time evolution of the layer thicknesses in the isolated tracer column experiments for four different initial positions of the column. The circled asterisks represent the thickness measured from the model outputs, while the plain lines represent the theoretical thickness inferred from Eq. (14). The simple linear scaling is a good estimation of the thinning of the layers.

$(m=1)$ modes start to grow according to Nguyen et al.'s (2012) linear stability analysis. The sum of the energy of modes $m=1$ and $m=2$ represents $95 \%$ of the total perturbation energy. A separate projection of the QGPV perturbation from the core and the lobes on azimuthal modes (not shown) confirms the preferential growth of mode 1 in the core and mode 2 in the lobes. Layering starts to develop during this linear stage and is obvious in the vertical profiles of high-pass filtered QGPV in Fig. 12d. Note also that at that stage, layering is much weaker at the vortex rotation axis, where no vertical shear of azimuthal velocity exists, confirming the key role of the latter in the layering process.

From days 100 to 170 (stage B), mode $m=1$ starts to decay while mode $m=2$ keeps on growing, corresponding to the growth of "spiral arms." Even though the growth of mode 2 during stage B is still nearly exponential, the ratio of QGPV perturbation to mean QGPV becomes too large to be referred to as linear instability, and we therefore chose to differentiate stages $\mathrm{A}$ and $\mathrm{B}$. The mode $m=2$ instability eventually breaks, as the growing spiral arms detach into two satellite vortices.

From day 170 until the end of the experiment, the lobes and core seem to follow a different evolution: while most of the negative QGPV remains coherent in the vortex core, as the latter restabilizes into an axisymmetric feature surrounded by smaller satellite vortices, the positive QGPV in the lobes is fragmented into thin stirred filaments. At that stage, layering is obvious in the vertical QGPV section as well as in the vertical high-pass filtered QGPV profiles in Figs. 12g and 12h. This last stage is obvious in the modal analysis as a collapse of the low azimuthal modes, associated with an increase of small-scale energy as the vortex is surrounded by turbulent-like variability.

As pointed out by Smith and Ferrari (2009), even though QGPV is an active tracer whose variability will affect the velocity field, it is a Lagrangian tracer, which is materially conserved along an isopycnal path, and will thus undergo a similar stirring as the passive tracer discussed in section 4. A parallel can be drawn between the passive tracer simulations and the nonlinear QG simulations. It was shown in section 4 that the velocity shear of the meddy would produce sharp vertical gradients provided the existence of initial azimuthal gradients. For layering to form in the QG model, it is thus necessary to introduce some azimuthal QGPV gradients. As described above, during the linear phase of destabilization, mode- 1 and mode- 2 perturbations simultaneously grow, breaking the vortex axisymmetry: as the mode $m=1$ perturbation induces a translation of the eddy core, the mode $m=2$ propagates around the lobes, introducing a shift between the core and the lobes and providing the necessary azimuthal variability for layering to occur. The preferential development of layering near-critical layers noted by Hua et al. (2013) can then be explained by the preferential growth of the unstable modes near the critical layers (Nguyen et al. 2012), resulting in more available azimuthal variability to be transformed in vertical gradients.

Once the unstable mode $m=2$ has detached, the vortex enters the nonlinear regime. The increase of layering near the lobes can also be discussed in terms of QGPV stirring. As shown in Figs. 12a and 12b, after the detachment of the spiral arms, the vortex core tends to reaxisymmetrize, while the positive $\mathrm{PV}$ lobes are fragmented into numerous filaments. This nearly turbulent state brings an intense azimuthal variability in the lobes, 


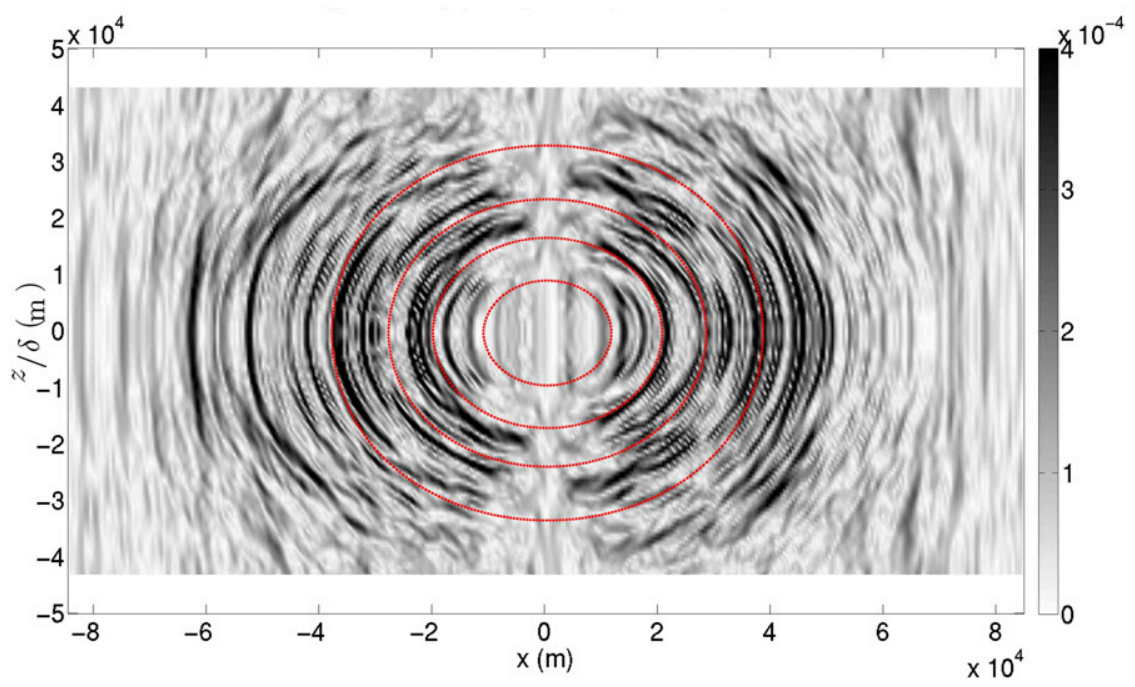

FIG. 11. Radial tracer gradient in stretched coordinates after 22 days of integration of the tracer model. The $z$ axis was stretched by the vortex aspect ratio. Layers are concentric rather than properly vertically stacked.

while the restabilized vortex core is nearly homogeneous and still has a strong and coherent baroclinic velocity signature. The intense QGPV horizontal gradients in the lobes are stirred by the vertically sheared velocity field, and the vertical variance cascade is enhanced. Note that unlike during the linear stage of destabilization, layering is also obvious in the central region of the lobes. This can be attributed to the turbulence inside the lobes that tends to homogenize the layering all over the meddy.

The fact that layering occurs preferentially in the vortex lobes and not in the core can be interpreted as the result of two joint effects: first the intensity of the azimuthal velocity vertical shear, which was shown to turn azimuthal gradients into vertical gradients is much more intense in the lobes (Figs. 2b,c); second, the distinctive fate of the core and lobes after the destabilization of the vortex leads to much more available azimuthal gradients in the lobes to be transformed into vertical gradients.

\section{Summary and discussion}

\section{a. Summary}

Based on a variety of frameworks, this study showed that tracer stirring was an efficient process to generate layering in the periphery of meddies and likely of vortex lenses in general.

Analysis of in situ data inside a meddy showed that layering was intense in terms of temperature and salinity, but that density-compensating effects of the latter two result in a weak signature in density, in agreement with Song et al.'s (2011) earlier results.
Outputs from a PE model showed very similar results. This is the first time to our knowledge that the layering phenomenon is modeled in this framework and that the density-compensating effects of temperature and salinity within these layers is observed in a numerical model. The use of the same diffusion coefficient for temperature and salinity in the model forbids any doublediffusive process to take place and might justify the enhanced density compensation in the model compared to real ocean data. It also suggests that, even though double-diffusive processes may impact layering, it is not a necessary condition for the latter to start developing and for the vertical variance cascade to occur. The contrasting behavior of spice (which showed intense layering) and density (which showed very little) in both the in situ data and PE model in the absence of doublediffusive processes suggested that layering can be triggered by along-isopycnal tracer stirring alone.

The latter hypothesis was investigated by means of a tracer advection model that showed that the vertically sheared velocity field associated with a Lorentzian vortex lens was efficient to generate strong layering from an initially barotropic tracer distribution. Sensitivity experiments, as well as a physical analysis of the tracer and tracer gradient evolution equations, showed that the tracer vertical gradient was linked to the initial azimuthal gradient through the rate of vertical shear of the azimuthal velocity. The time evolution of the ratio of vertical to azimuthal variability was also shown to depend only on the vertical shear of the azimuthal velocity. At a given radial position from the vortex center, a simple linear scaling was proposed and represents an 

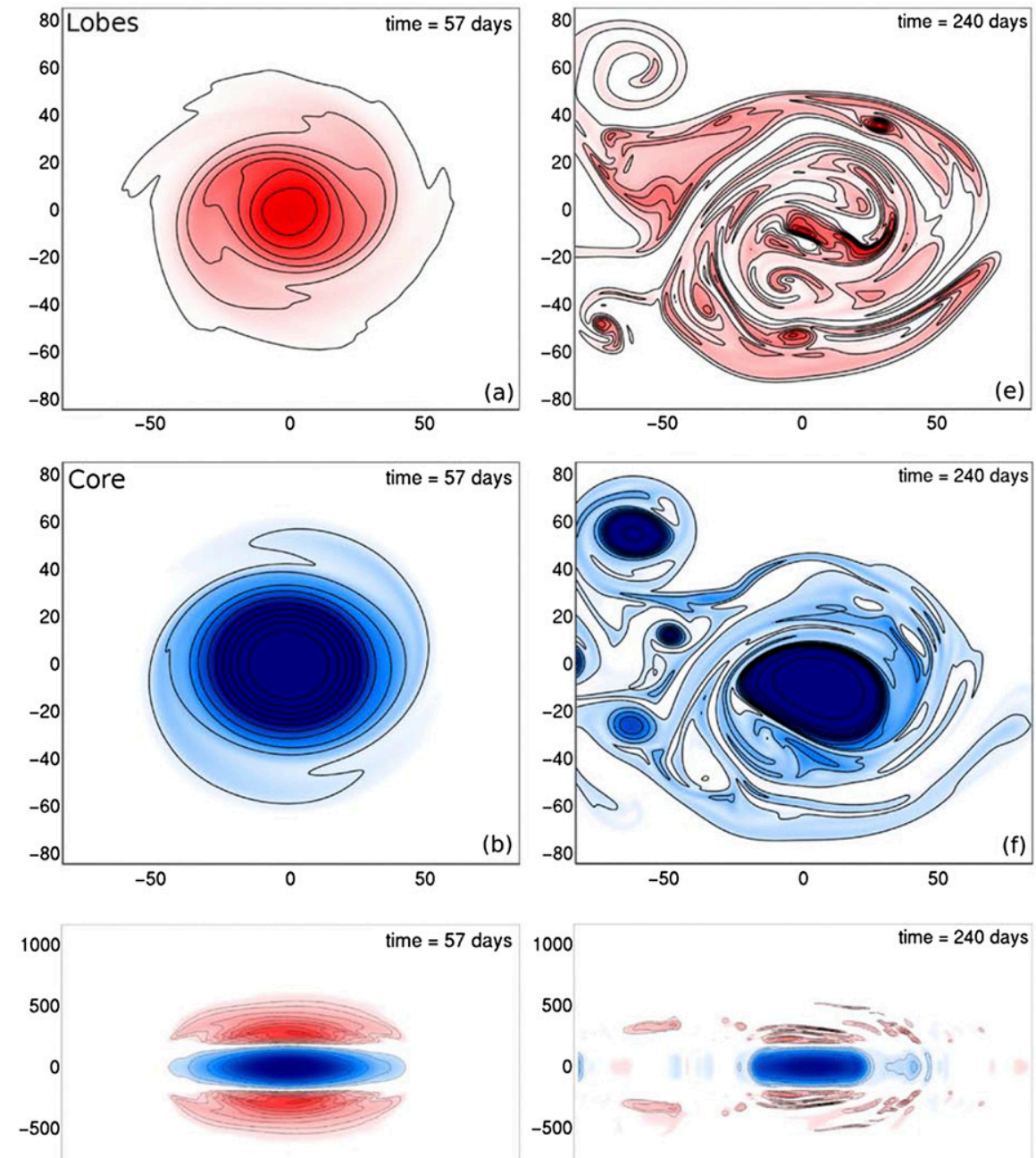

ime $=57$ days

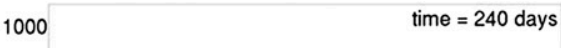

$-1000$

(c) -1000

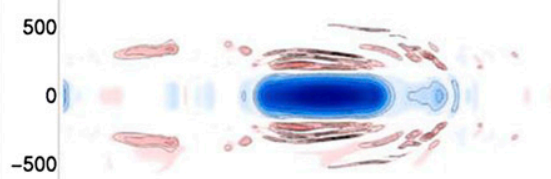

$-1000$

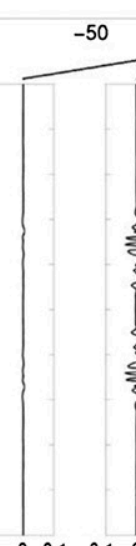

(g)
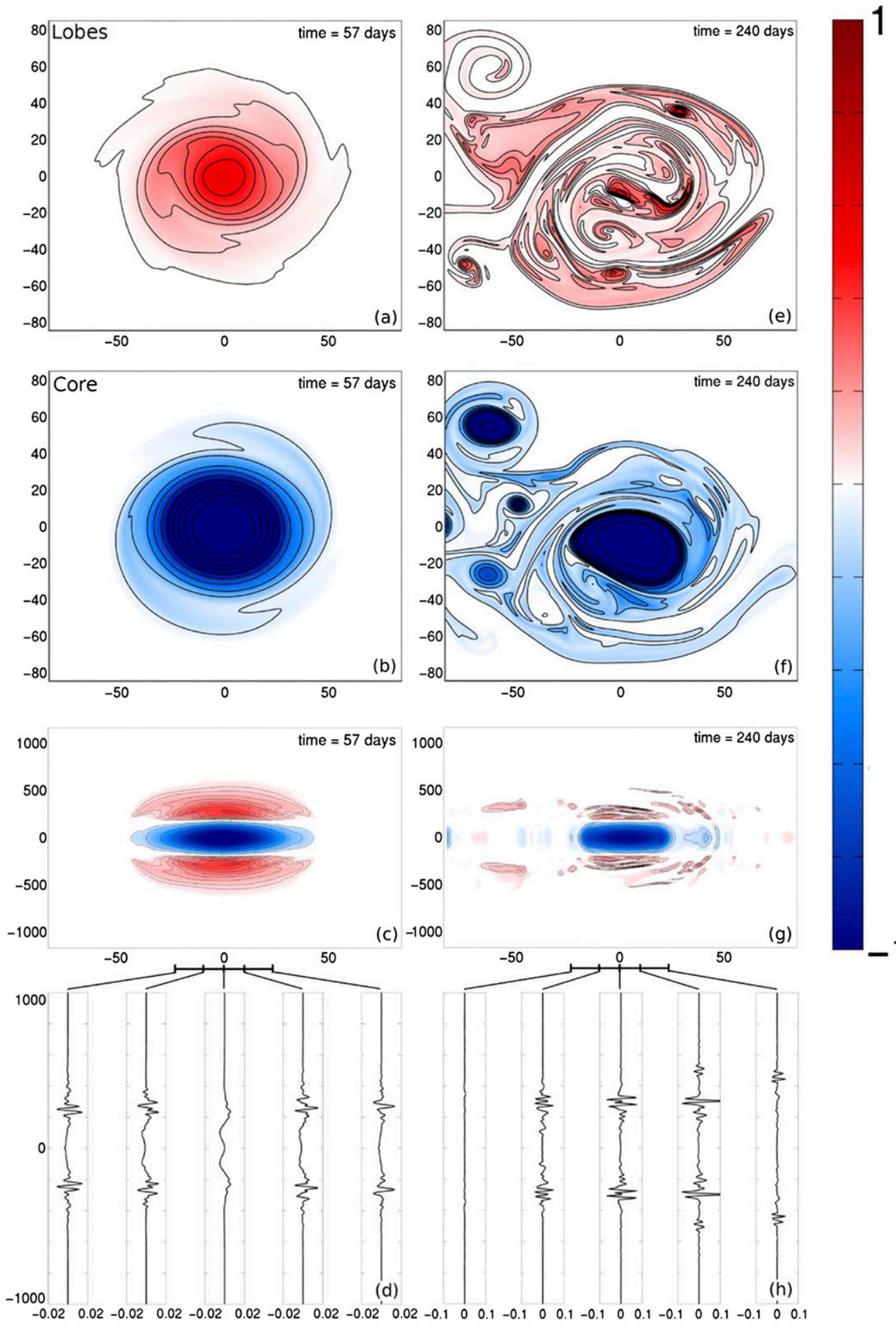

FIG. 12. Snapshots from the nonlinear QG simulations after (left) 57 and (right) 240 days of model run. (a),(e) QGPV horizontal section in the eddy lobes. (b),(f) QGPV horizontal section in the eddy core. (c),(g) QGPV vertical section. (d),(h) Vertical profiles of high-pass filtered QGPV perturbation at five distinct locations. Layering starts to develop during the linear stage of destabilization. It is greatly enhanced once the vortex lobes have reached a nearly turbulent state. 


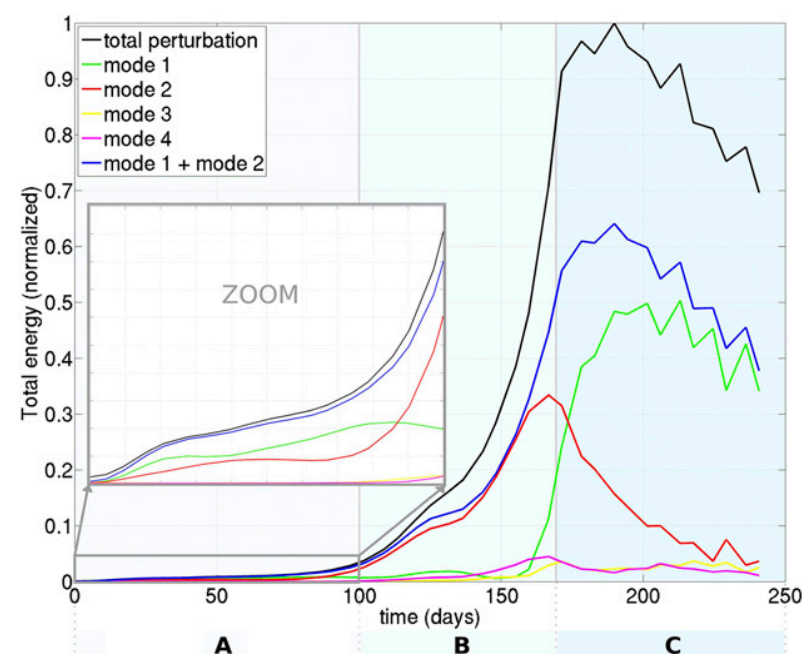

FIG. 13. Time evolution of the perturbation energy in the nonlinear QG simulations. The black line is the total perturbation energy while the green, red, yellow, purple, and blue lines are, respectively, the energy of azimuthal modes $1,2,3$, and 4 and the sum of modes 1 and 2. Most of the total perturbation energy is contained by the first two modes.

efficient estimate of the thinning down of the layers with time in the case of tracer advection by a steady mean flow. The layer thickness reads $\epsilon=\left(2 L_{\phi}\right) / \sigma t$, where $L_{\phi}$ is the initial radius of the tracer column, $\sigma$ is the rate of vertical shear of the azimuthal velocity, and $t$ is the time.

After this rationalization of the transfer of horizontal azimuthal variability into vertical variability in a stationary flow, a further interpretation of Hua et al.'s (2013) results was proposed in the QG framework. It showed that the role of the critical layers emerges from the favored formation of PVA perturbations near the critical layers, feeding the vortex velocity field with the necessary azimuthal variability for layering to occur. The role of baroclinic instability in the early stage of layering is thus likely to be linked to the breaking of axisymmetry as the perturbations start to grow. Finally, strong nonlinear interactions in the vortex lobes after the total destabilization of the vortex leads to the fragmentation and filamentation of the positive PV lobes, while the vortex core tends to reaxisymmetrize. This PV fragmentation is the source of azimuthal variability leading to enhanced layering after a sufficiently long time.

In this study, we also note that the existence of a nonuniform radial gradient of azimuthal velocity results in the formation of horizontally stacked layers on the side of the meddy, or more generally, that the layers are likely to be concentric rather than properly vertically stacked.

From a more general point of view, this study shows that a meddy velocity field is able to transform any azimuthal variability into vertical variability.

\section{b. Discussion and perspectives}

Here, we focused on the formation of layering and on the variance cascade down to scales of $O(10) \mathrm{m}$ and did not investigate the physical processes responsible for the halting of this cascade at small scale. In the frictionless tracer advection model we used, the cascade is only limited by the vertical grid resolution, while in the linear scaling nothing acts to stop the scale cascade. For instance, for an initially 5-km-wide tracer column, the scaling predicts that a vertical shear of $5 \times 10^{-4} \mathrm{~s}^{-1}$ will generate 1-m-thick layers after 100 days. Such small layers are unlikely to be observed around real ocean meddies, where the layering scales range between 15 and $100 \mathrm{~m}$. Some process must thus be invoked to explain the limitation of the vertical variance cascade. Indeed, even though the good agreement between our numerical results and in situ data suggests that stirring is an efficient process in layering formation at the vertical scales considered here (10 to $100 \mathrm{~m}$ ), under the $O$ (10) m vertical scale and at time scales comparable with a meddy life time, diffusive processes might become important and act to mix the tracer and block the variance cascade. Even though double diffusion was shown not to be necessary in the formation stage of layering, it is expected to play an important role at these small vertical scales (that are not solved by the models), as shown by Ruddick (1992) and Ruddick and Walsh (1995). As noted by Song et al. (2011), a full understanding of layering dynamics and its finescale limitations should include vertical diffusivity, whether turbulent dissipation, double diffusion, or other possible mechanisms such as diffusive instability or nonhydrostatic effects.

Note that in the dynamical models used here (PE and QG), the horizontal gradients necessary for the generation of vertical gradients is intrinsic to the meddy in the sense that it arises from instability; the vortex generates its own variability that is then transformed into vertical variability. But one can think of external variability as a source of layering. As they drift through the eastern North Atlantic, meddies are surrounded by submesoscale turbulence that will interact with the vertically sheared azimuthal flow and could thus permanently trigger layering.

Finally, one should note that not only intrathermocline lenses such as meddies are baroclinic vortices. As the process proposed here is entirely based on stirring effects by a vertically sheared azimuthal flow, one can suppose that layering could also be observed below any surface-intensified vortex, where the vorticity distribution is strongly baroclinic. The layering process, which seems to play a key role in the decay of meddies and 
thermodynamic dissipation toward the ocean (Ruddick et al. 2010), might thus be at work under most energetic eddies and would require further investigation both in observational and numerical frameworks.

Acknowledgments. This study was supported by the SIMILA (SIMI 5-6, 2011) and OLA (PDOC 2011) projects granted by the French National Agency for Research (ANR). The OLA project was initiated and led by Pr. B. Lien Hua, who died on 26 November 2012. This study would not have been possible without her insightful and pioneering work on vortex dynamics. The authors dedicate this paper to her memory. They are also grateful to Patrice Klein and Xavier Carton for their useful comments and suggestions during this study.

\section{REFERENCES}

Aiki, H., and T. Yamagata, 2006: Energetics of the layer-thickness form drag based on an integral identity. Ocean Sci., 2, 161-171, doi:10.5194/os-2-161-2006.

_ J. P. Matthews, and K. G. Lamb, 2011: Modeling and energetics of tidally generated wave trains in the Lombok Strait: Impact of the Indonesian Throughflow. J. Geophys. Res., 116, C03023, doi:10.1029/2010JC006589.

Armi, L., and W. Zenk, 1984: Large lenses of highly saline Mediterranean water. J. Phys. Oceanogr., 14, 1560-1576, doi:10.1175/1520-0485(1984)014<1560:LLOHSM>2.0.CO;2.

, D. Hebert, N. Oakey, J. F. Price, P. L. Richardson, H. T. Rossby, and B. Ruddick, 1989: Two years in the life of a Mediterranean salt lens. J. Phys. Oceanogr., 19, 354-370, doi:10.1175/1520-0485(1989)019<0354:TYITLO > 2.0.CO;2.

Balmforth, N. J., S. G. L. Smith, and W. R. Young, 1998: Dynamics of interfaces and layers in a stratified turbulent fluid. J. Fluid Mech., 355, 329-358, doi:10.1017/S0022112097007970.

Barbosa Aguiar, A. C., Á. Peliz, and X. Carton, 2013: A census of meddies in a long-term high-resolution simulation. Prog. Oceanogr., 116, 80-94, doi:10.1016/j.pocean.2013.06.016.

Beckmann, A., and R. H. Käse, 1989: Numerical simulation of the movement of a Mediterranean water lens. Geophys. Res. Lett., 16, 65-68, doi:10.1029/GL016i001p00065.

Biescas, B., V. Sallarès, J. L. Pelegrí, F. Machín, R. Carbonell, G. Buffett, J. J. Dañobeitia, and A. Calahorrano, 2008: Imaging meddy finestructure using multichannel seismic reflection data. Geophys. Res. Lett., 35, L11609, doi:10.1029/ 2008GL033971.

_ B. R. Ruddick, M. R. Nedimovic, V. Sallarès, G. Bornstein, and J. F. Mojica, 2014: Recovery of temperature, salinity, and potential density from ocean reflectivity. J. Geophys. Res. Oceans, 119, 3171-3184, doi:10.1002/2013JC009662.

Bower, A. S., L. Armi, and I. Ambar, 1997: Lagrangian observations of meddy formation during a Mediterranean Undercurrent seeding experiment. J. Phys. Oceanogr., 27, 2545-2575, doi:10.1175/1520-0485(1997)027<2545: LOOMFD $>2.0 . \mathrm{CO} ; 2$.

Carton, X., L. Chérubin, J. Paillet, Y. Morel, A. Serpette, and B. Le Cann, 2002: Meddy coupling with a deep cyclone in the Gulf of Cadiz. J. Mar. Syst., 32, 13-42, doi:10.1016/S0924-7963(02)00028-3.

—, N. Daniault, J. Alves, L. Cherubin, and I. Ambar, 2010: Meddy dynamics and interaction with neighboring eddies southwest of Portugal: Observations and modeling. J. Geophys. Res., 115, C06017, doi:10.1029/2009JC005646.

Dukowicz, J. K., and R. D. Smith, 1994: Implicit free-surface method for the Bryan-Cox-Semtner ocean model. J. Geophys. Res., 99, 7991-8014, doi:10.1029/93JC03455.

Haynes, P., and J. Anglade, 1997: The vertical-scale cascade in atmospheric tracers due to large-scale differential advection. J. Atmos. Sci., 54, 1121-1136, doi:10.1175/1520-0469(1997)054<1121: TVSCIA $>2.0 . \mathrm{CO} ; 2$.

Hebert, D., N. Oakey, and B. Ruddick, 1990: Evolution of a Mediterranean salt lens: Scalar properties. J. Phys. Oceanogr., 20, 1468-1483, doi:10.1175/1520-0485(1990)020<1468: EOAMSL $>2.0 . \mathrm{CO} ; 2$.

Hobbs, R. W., 2007: Geophysical oceanography (GO): A new tool to understand the thermal structure and dynamics of oceans. AAPG European Region Newsletter, Vol. 2, AAPG European Region, Imperial College, London, United Kingdom, 7. [Available online at http://archives.aapg.org/europe/newsletters/2007/ 06jun/06jun07europe.pdf.]

Hua, B. L., and D. B. Haidvogel, 1986: Numerical simulations of the vertical structure of quasi-geostrophic turbulence. J. Atmos. Sci., 43, 2923-2936, doi:10.1175/1520-0469(1986)043<2923: NSOTVS $>2.0 . \mathrm{CO} ; 2$.

_ C. Ménesguen, S. Le Gentil, R. Schopp, B. Marsset, and H. Aiki, 2013: Layering and turbulence surrounding an anticyclonic oceanic vortex: In situ observations and quasigeostrophic numerical simulations. J. Fluid Mech., 731, 418442, doi:10.1017/jfm.2013.369.

Jungclaus, J. H., 1999: A three-dimensional simulation of the formation of anticyclonic lenses (meddies) by the instability of an intermediate depth boundary current. J. Phys. Oceanogr., 29, 1579-1598, doi:10.1175/1520-0485(1999)029<1579: ATDSOT $>2.0 . \mathrm{CO} ; 2$.

Klein, P., A. M. Treguier, and B. L. Hua, 1998: Three-dimensional stirring of thermohaline fronts. J. Mar. Res., 56, 589-612, doi:10.1357/002224098765213595.

MacVean, M. K., and J. D. Woods, 1980: Redistribution of scalars during upper ocean frontogenesis: A numerical model. Quart. J. Roy. Meteor. Soc., 106, 293-311, doi:10.1002/qj.49710644805.

Ménesguen, C., B. L. Hua, C. Papenberg, D. Klaeschen, L. Geli, and R. Hobbs, 2009: Effect of bandwidth on seismic imaging of rotating stratified turbulence surrounding an anticyclonic eddy from field data and numerical simulations. Geophys. Res. Lett., 36, L00D05, doi:10.1029/2009GL039951.

Morel, Y., and J. McWilliams, 1997: Evolution of isolated interior vortices in the ocean. J. Phys. Oceanogr., 27, 727-748, doi:10.1175/1520-0485(1997)027<0727:EOIIVI>2.0.CO;2.

Munk, W., 1981: Internal waves and small-scale processes. Evolution of Physical Oceanography: Scientific Surveys in Honor of Henry Stommel, B. A. Warren and C. Wunsch, Eds., MIT Press, 264-291.

Nandi, P., W. S. Holbrook, S. Pearse, P. Páramo, and R. W. Schmitt, 2004: Seismic reflection imaging of water mass boundaries in the Norwegian Sea. Geophys. Res. Lett., 31, L23311, doi:10.1029/2004GL021325.

Nguyen, H. Y., B. L. Hua, R. Schopp, and X. Carton, 2012: Slow quasigeostrophic unstable modes of a lens vortex in a continuously stratified flow. Geophys. Astrophys. Fluid Dyn., 106, 305-319, doi:10.1080/03091929.2011.620568.

Paillet, J., B. Le Cann, X. Carton, Y. Morel, and A. Serpette, 2002: Dynamics and evolution of a northern meddy. J. Phys. Oceanogr., 32, 55-79, doi:10.1175/1520-0485(2002)032<0055: DAEOAN $>2.0 . \mathrm{CO} ; 2$. 
Pingree, R. D., and B. Le Cann, 1993: Structure of a meddy (Bobby 92) southeast of the Azores. Deep-Sea Res. I, 40, 2077-2103, doi:10.1016/0967-0637(93)90046-6.

Pinheiro, L. M., H. Song, B. Ruddick, J. Dubert, I. Ambar, K. Mustafa, and R. Bezerra, 2010: Detailed 2-D imaging of the Mediterranean outflow and meddies off W Iberia from multichannel seismic data. J. Mar. Syst., 79, 89-100, doi:10.1016/ j.jmarsys.2009.07.004.

Prater, M. D., and T. B. Sanford, 1994: A meddy off Cape St. Vincent. Part I: Description. J. Phys. Oceanogr., 24, 1572-1586, doi:10.1175/1520-0485(1994)024<1572:AMOCSV>2.0.CO;2.

Quentel, E., X. Carton, M.-A. Gutscher, and R. Hobbs, 2010: Detecting and characterizing mesoscale and submesoscale structures of Mediterranean water from joint seismic and hydrographic measurements in the Gulf of Cadiz. Geophys. Res. Lett., 37, L06604, doi:10.1029/2010GL042766.

Rhines, P. B., and W. R. Young, 1983: How rapidly is a passive scalar mixed within closed streamlines? J. Fluid Mech., 133, 133-145, doi:10.1017/S0022112083001822.

Richardson, P. L., A. S. Bower, and W. Zenk, 2000: A census of meddies tracked by floats. Prog. Oceanogr., 45, 209-250, doi:10.1016/S0079-6611(99)00053-1.

Ruddick, B., 1992: Intrusive mixing in a Mediterranean salt lensIntrusion slopes and dynamical mechanisms. J. Phys. Oceanogr., 22, 1274-1285, doi:10.1175/1520-0485(1992)022<1274: IMIAMS $>2.0 . \mathrm{CO} ; 2$.

, and D. Hebert, 1988: The mixing of Meddy Sharon. SmallScale Turbulence and Mixing in the Ocean, J. C. J. Nihoul and B. M. Jamart, Ed., Elsevier Oceanography Series, Vol. 46, Elsevier, 249-261, doi:10.1016/S0422-9894(08)70551-8.
— , and D. Walsh, 1995: Observations of the density perturbations which drive thermohaline intrusions. Double-Diffusive Convection, Geophys. Monogr., Vol. 94, Amer. Geophys. Union, 329-334.

- , H. Song, C. Dong, and L. Pinheiro, 2009: Water column seismic images as maps of temperature gradient. Oceanography, 22, 192-205, doi:10.5670/oceanog.2009.19.

_ N. S. Oakey, and D. Hebert, 2010: Measuring lateral heat flux across a thermohaline front: A model and observational test. J. Mar. Res., 68, 523-539, doi:10.1357/002224010794657146.

Schultz-Tokos, K. L., and T. Rossby, 1991: Kinematics and dynamics of a Mediterranean salt lens. J. Phys. Oceanogr., 21, 879-892, doi:10.1175/1520-0485(1991)021<0879:KADOAM>2.0.CO;2.

- - H. H. Hinrichsen, and W. Zenk, 1994: Merging and migration of two meddies. J. Phys. Oceanogr., 24, 2129-2141, doi:10.1175/1520-0485(1994)024<2129:MAMOTM>2.0.CO;2.

Smith, K. S., and R. Ferrari, 2009: The production and dissipation of compensated thermohaline variance by mesoscale stirring. J. Phys. Oceanogr., 39, 2477, doi:10.1175/2009JPO4103.1.

Song, H., L. M. Pinheiro, B. Ruddick, and F. C. Teixeira, 2011: Meddy, spiral arms, and mixing mechanisms viewed by seismic imaging in the Tagus Abyssal Plain (SW Iberia). J. Mar. Res., 69, 4-6, doi:10.1357/002224011799849309.

Veronis, G., 1972: Properties of seawater defined by temperature, salinity, and pressure. J. Mar. Res., 30, 227-255.

Weiss, J., 1991: The dynamics of enstrophy transfer in twodimensional hydrodynamics. Physica D, 48, 273-294, doi:10.1016/ 0167-2789(91)90088-Q.

Woods, J. D., R. Onken, and J. Fischer, 1986: Thermohaline intrusions created isopycnically at oceanic fronts are inclined to isopycnals. Nature, 322, 446-449, doi:10.1038/322446a0. 